Supporting Information

\title{
Highly Compressive Boron Nitride Nanotube Aerogels Reinforced with Reduced Graphene Oxide
}

Mingmei Wang ${ }^{a, b, \dagger}$, Tao Zhang ${ }^{a, \dagger}$, Dasha Mao ${ }^{a, c}$, Yimin Yao $^{a, c}$, Xiangliang Zeng,

Linlin Ren ${ }^{a}$, Qiran Cai ${ }^{d}$, Srikanth Mateti ${ }^{d}$, Lu Hua Li ${ }^{d}$, Xiaoliang Zeng ${ }^{a^{*}}$, Guoping $D u^{b^{*}}$, Rong Sun ${ }^{a^{*}}$, Ying Chen ${ }^{d^{*}}$, Jian-Bin Xu $u^{e}$ and Ching-Ping Wong $f$

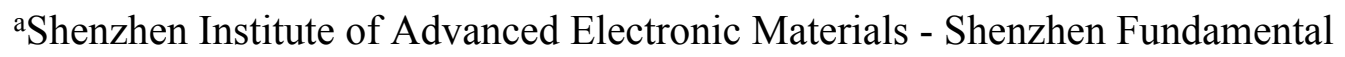

Research Institutions, Shenzhen Institutes of Advanced Technology, Chinese

Academy of Sciences, Shenzhen 518055, China

${ }^{\mathrm{b} S c h o o l ~ o f ~ M a t e r i a l s ~ S c i e n c e ~ a n d ~ E n g i n e e r i n g, ~ N a n c h a n g ~ U n i v e r s i t y, ~ N a n c h a n g ~}$ 330031, China

'Shenzhen College of Advanced Technology, University of Chinese Academy of Sciences, Shenzhen 518055, China

dinstitute for Frontier Materials, Deakin University, Waurn Ponds, Victoria 3216, Australia.

eDepartment of Electronics Engineering, The Chinese University of Hong Kong, Hong Kong 999077, China

fSchool of Materials Science and Engineering, Georgia Institute of Technology, Atlanta, Georgia 30332, United States

$\dagger$ These authors contributed equally

*To whom correspondence should be addressed. E-mail: xl.zeng@siat.ac.cn (X.Z.); guopingdu@ncu.edu.cn (G.D.); rong.sun@siat.ac.cn (R.S.); $\underline{\text { ian.chen@,deakin.edu.au }}$ (Y.C.) 
Contents

Figure S1. AFM image and height profile of GO nanosheets.

Figure S2. The microscopic morphology of pure rGO aerogel.

Figure S3. TEM images of the BNNTs/rGO hybrids.

Figure S4. TGA curves of pristine BNNTs, rGO and BN30 (composite aerogel with 30 wt $\%$ BNNTs loading).

Figure S5. The stress-strain curves of BNNTs/rGO hybrid aerogels before and after cycling 100 times at 50\% compression.

Figure S6. SEM images of BNNTs/rGO/PEG composites.

Figure S7. The enhancement efficiency of thermal conductivity for BNNTs/rGO/PEG composites as a function of BNNTs/rGO loadings.

Figure S8. The pictures of Hot Disk device.

Table S1. The elemental composition, atomic content and calculated $\mathrm{C} / \mathrm{O}$ ratio in sample GO, rGO and BNNTs/rGO.

Table S2. The phase change temperature and phase change enthalpy of pure PEG, rGO/PEG, BNNTs-10/rGO/PEG, BNNTs-30/rGO/PEG and BNNTs-50/rGO/PEG. 


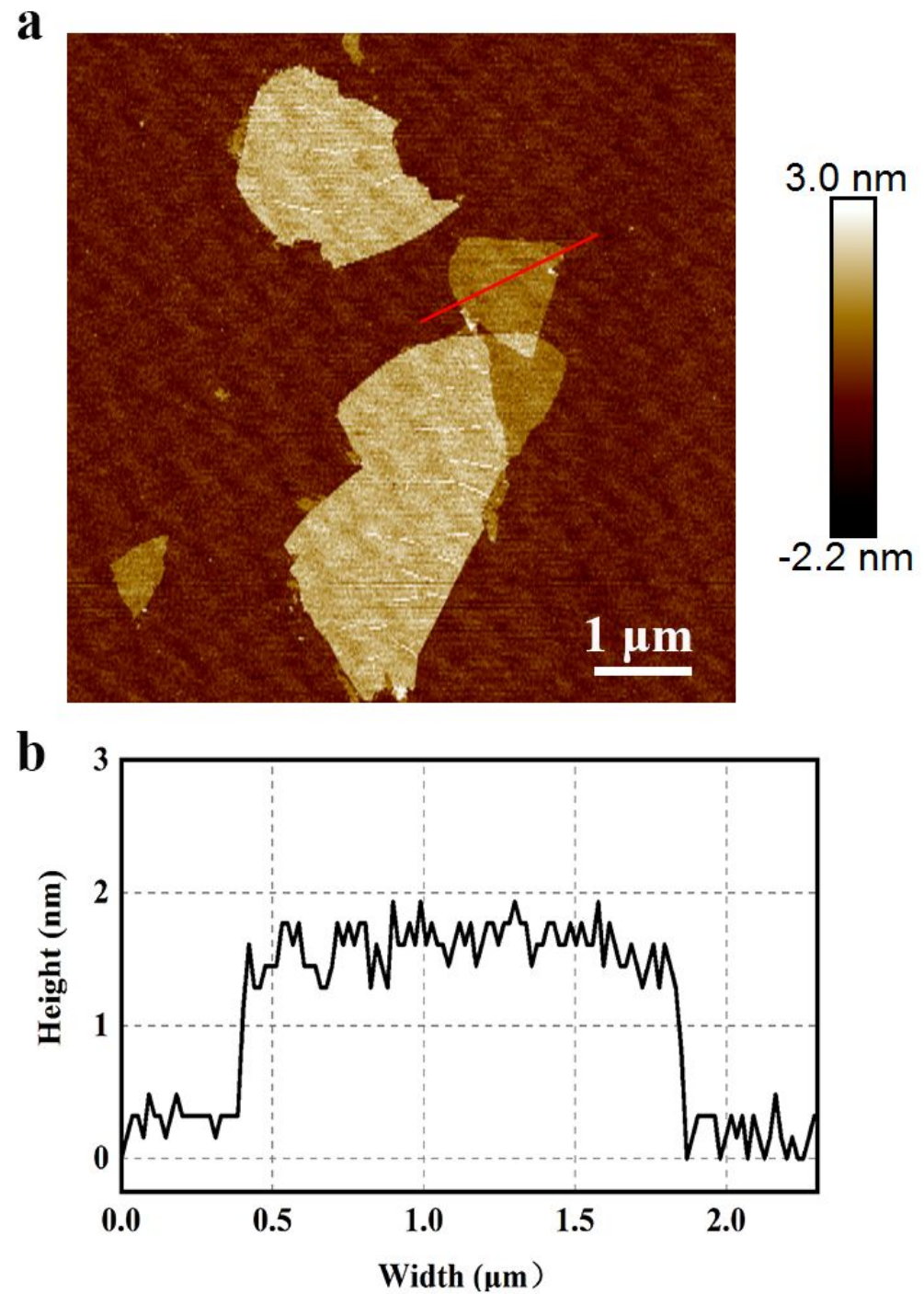

Figure S1. AFM image and height profile of GO nanosheets. 


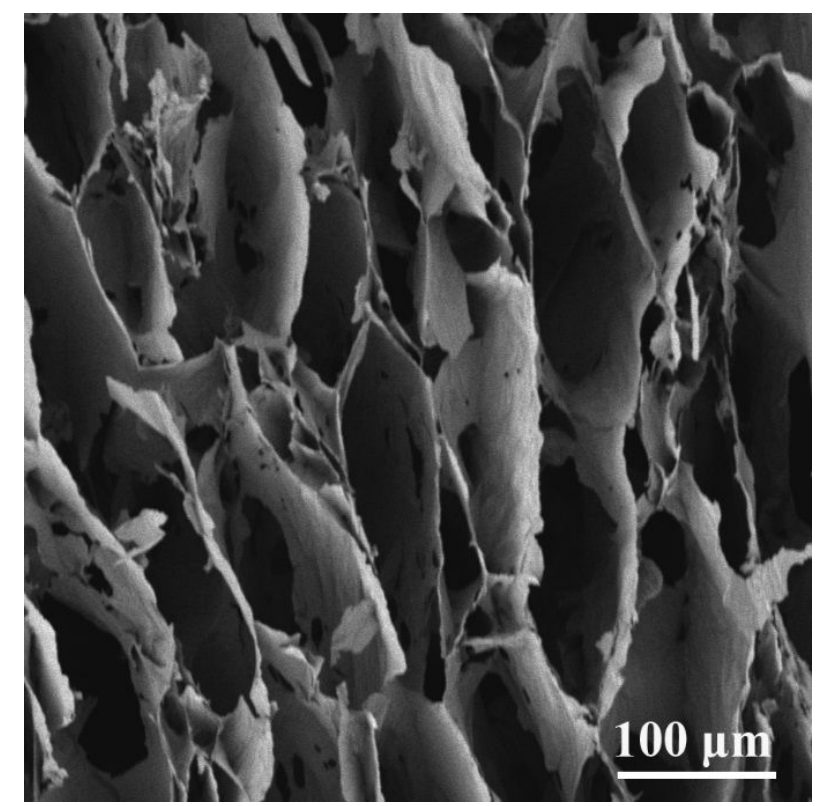

Figure S2. The microscopic morphology of pure rGO aerogel. 

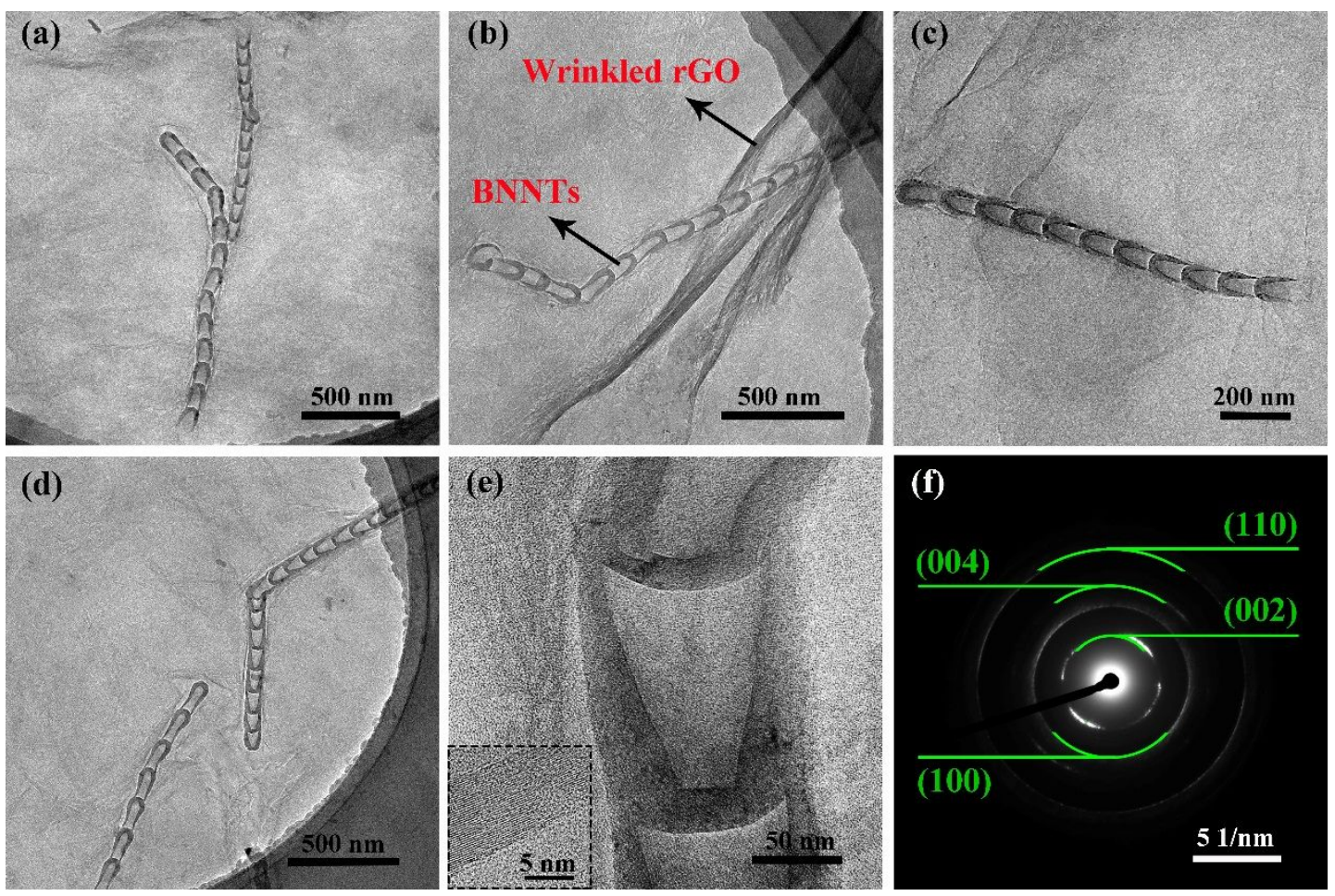

Figure S3. TEM images of the BNNTs/rGO hybrids. (a-d) The microscopic morphology of BNNTs/rGO hybrids. (e) TEM image of BNNT (inset: HRTEM image). (f) The SAED pattern of BNNT. 


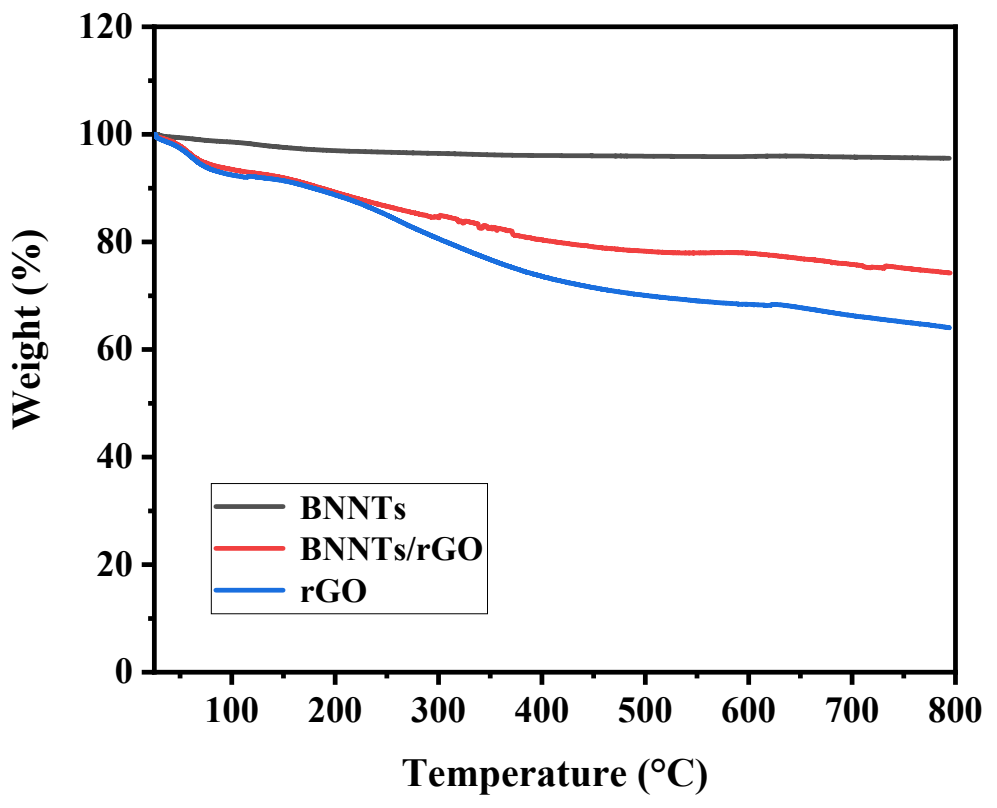

Figure S4. TGA curves of pristine BNNTs, rGO and BNNTs/rGO hybrid aerogel with $30 \mathrm{wt} \%$ BNNTs loading. 


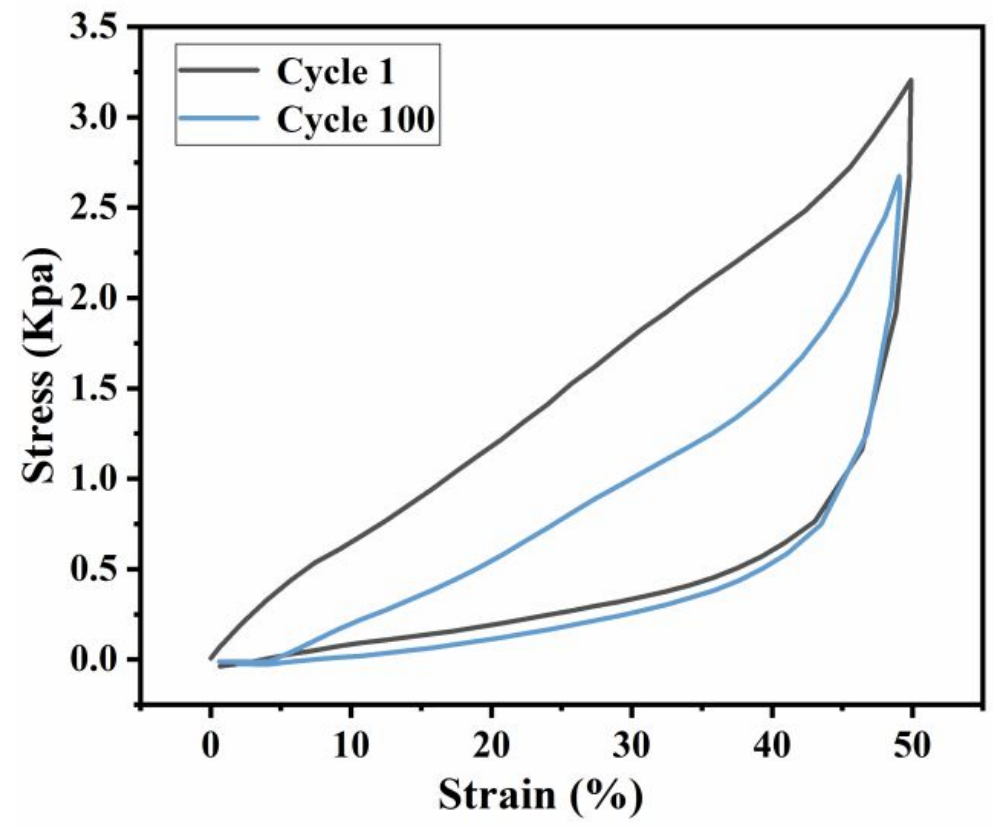

Figure S5. The comparision of stress-strain curves of the BNNTs/rGO hybrid aerogels before and after cycled 100 times at 50\% compression. 

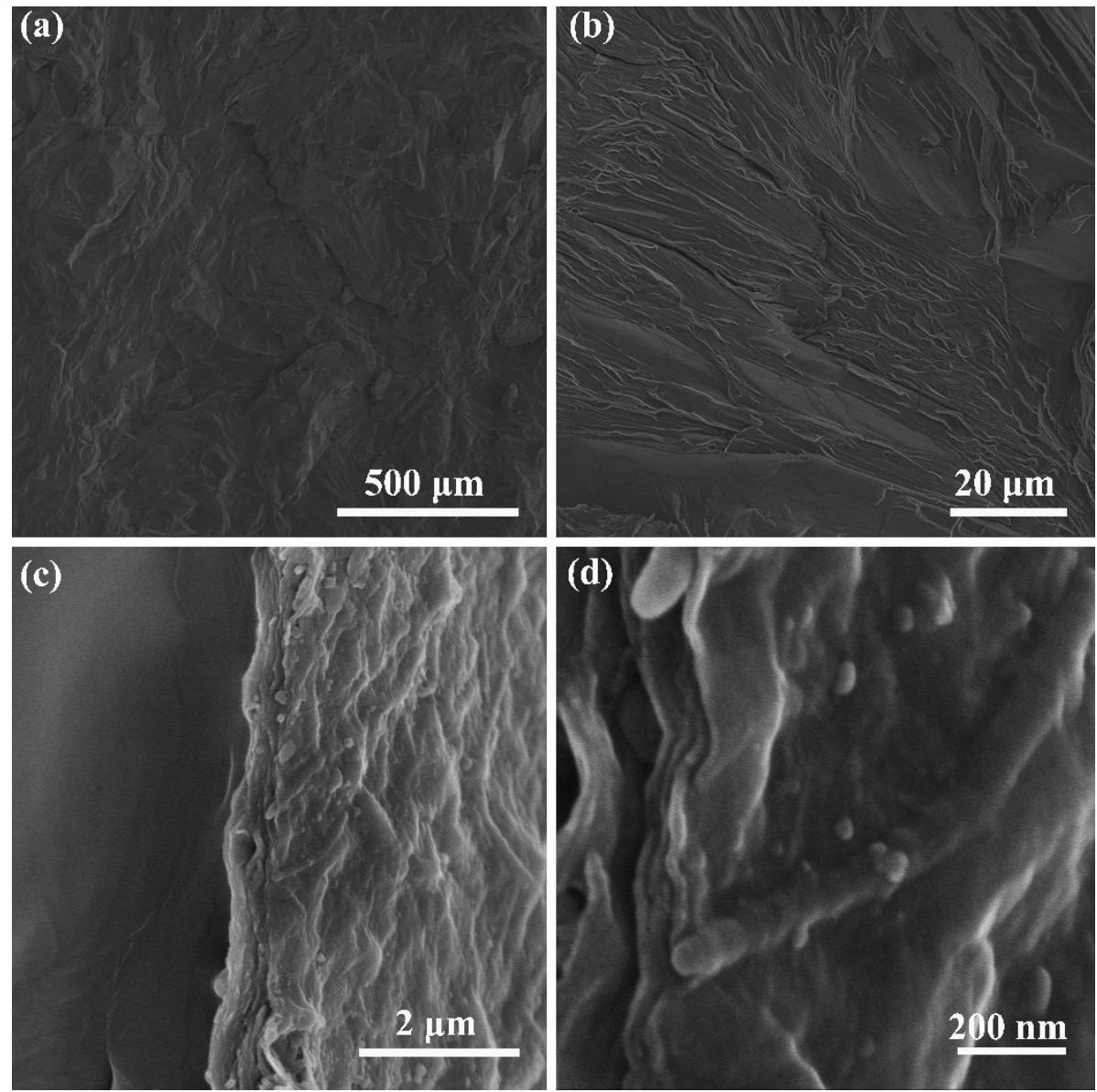

Figure S6. The SEM images of the BNNTs/rGO/PEG composites. (a) Lowmagnification SEM image of BNNTs/rGO/PEG composites. (b) The layered structure formed during PEG crystallization. (c-d) BNNTs at the fracture edge of BNNTs/rGO/PEG. 


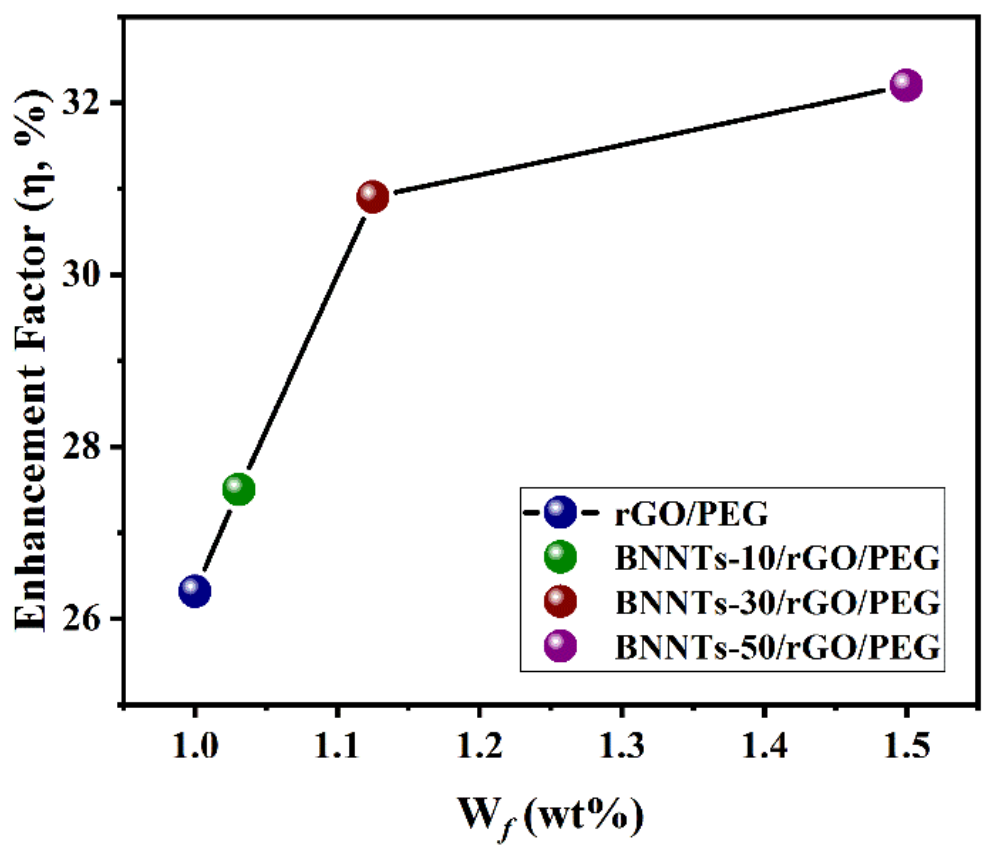

Figure S7. The enhancement efficiency of thermal conductivity for 3D BNNTs/rGO/PEG composites as a function of BNNTs/rGO loadings. 

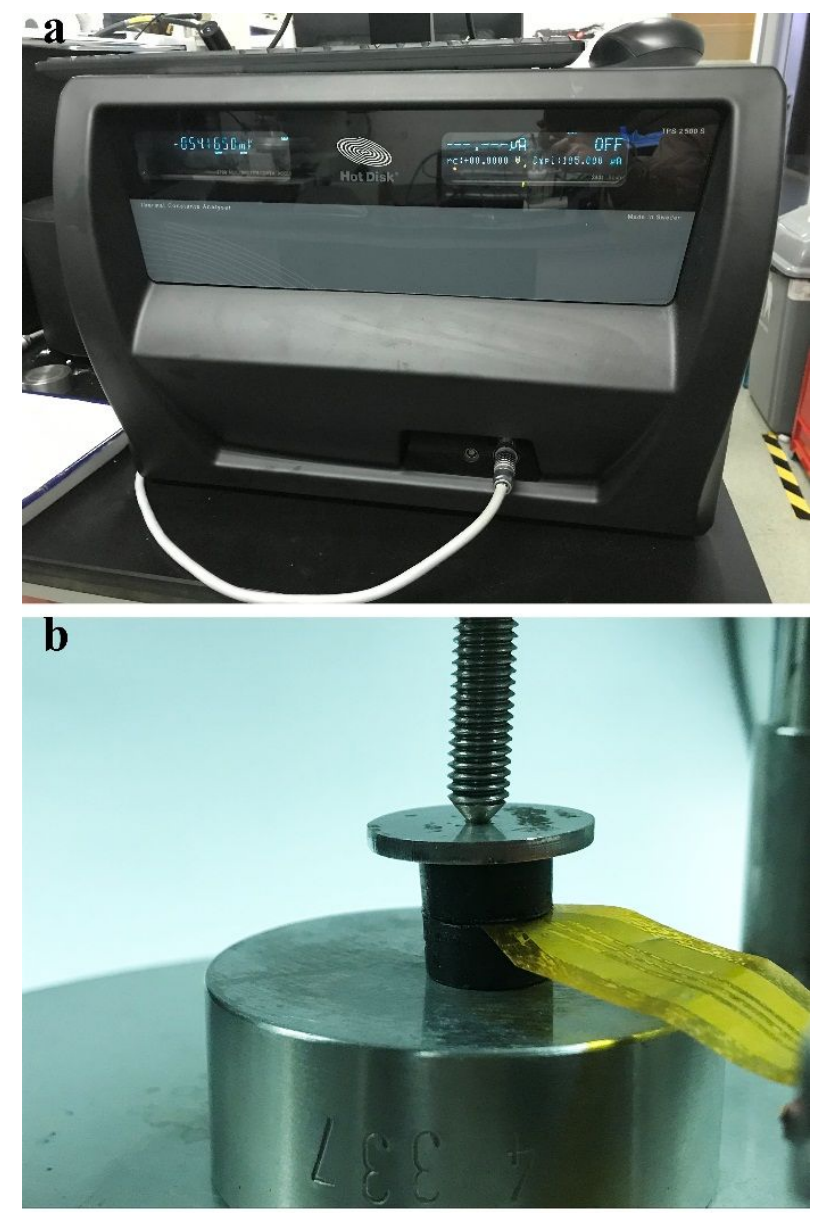

Figure S8. (a) The picture of Hot Disk device. (b) The picture during sample testing. 
Table S1. The elemental composition, atomic content and calculated $\mathrm{C} / \mathrm{O}$ ratio in sample GO, rGO and BNNTs/rGO.

\begin{tabular}{lcccccc}
\hline & Sample & B1s & C1s & N1s & O1s & C/O \\
\hline & GO & 0.00 & 34.82 & 1.11 & 31.87 & 1.09 \\
$\begin{array}{l}\text { Atomic } \\
(\%)\end{array}$ & rGO & 0.00 & 46.32 & 13.16 & 11.21 & 4.13 \\
\hline
\end{tabular}


Table S2. The phase change temperature and phase change enthalpy of pure PEG, rGO/PEG, BNNTs-10/rGO/PEG, BNNTs-30/rGO/PEG and BNNTs-50/rGO/PEG.

\begin{tabular}{ccccc}
\hline Sample & $\begin{array}{c}\text { Fusion } \\
\text { temperature } \\
\left({ }^{\circ} \mathbf{C}\right)\end{array}$ & $\begin{array}{c}\text { Fusion } \\
\text { enthalpy } \\
(\mathbf{J} / \mathbf{g})\end{array}$ & $\begin{array}{c}\text { Solidification } \\
\text { temperature } \\
\left({ }^{\circ} \mathbf{C}\right)\end{array}$ & $\begin{array}{c}\text { Solidification } \\
\text { enthalpy } \\
(\mathbf{J} / \mathbf{g})\end{array}$ \\
\hline PEG & 59.11 & 188.50 & 37.23 & 170.00 \\
rGO/PEG & 58.86 & 182.31 & 37.69 & 164.30 \\
BNNTs-10/rGO/PEG & 59.02 & 183.98 & 37.87 & 167.60 \\
BNNTs-30/rGO/PEG & 59.43 & 185.50 & 38.35 & 166.50 \\
BNNTs-50/rGO/PEG & 59.14 & 195.60 & 38.49 & 175.90 \\
\hline
\end{tabular}

